\title{
Evaluation of metabolic and hemodynamic alterations in the offspring of perinatally stressed rats
}

\author{
Mireille Toledo-Blas, M. Lopez-Martinez, P. Lopez-Sanchez, A. Guzman-Grenfell, A. Meneses- \\ Ortiz, Antonio Franco Vadillo
}

Laboratorio de Farmacologia Experimental Instituto Nacional de Perinatologia, Mexico

The present work allows clarifying the importance of the adverse environment during the first stages of development as a key factor for the predisposition to metabolic, hemodynamic and behavioral diseases in adulthood. This is a phenomenon also known as programming, which is defined as an adverse event in a critical and sensitive period of early life that will produce permanent effects on the structure, physiology and metabolism of the subject and that can extend to the following generations.

In the present work a programming model was designed where we worked with offspring of rats that were subjected to adverse events during pregnancy with a movement restriction model as prenatal stress and maternal separation as postnatal stress. The results showed that some metabolic markers such as triglycerides and total cholesterol (particularly LDL) increased significantly in animals subjected to chronic stress due to restriction to movement during pregnancy; combined with postnatal stress due to early maternal separation. On the other hand, no significant differences were found in hemodynamic variables. However, there are changes in the molecular expression of AT1, AT2 and PRR in the hypothalamus, with a significant increase in AT1 and PRR, in addition to a significant decrease in AT2. These markers are known to intervene in the regulation of vascular physiology. 